15 Vos R, Blondeau K, Vanaudenaerde BM, et al. Airway colonization and gastric aspiration after lung transplantation: do birds of a feather flock together? J Heart Lung Transplant 2008; 27: 843-849.

16 Gregson AL, Wang X, Weigt SS, et al. Interaction between Pseudomonas and CXC chemokines increases risk of bronchiolitis obliterans syndrome and death in lung transplantation. Am J Respir Crit Care Med 2013; 187: $518-526$

\title{
Chronic pulmonary disease with Mycobacterium abscessus complex is a biofilm infection
}

To the Editor:

Direct evidence of Mycobacterium abscessus complex (MABSC) biofilm in the human lung has not previously been demonstrated. Biofilms are microcolonies of bacteria, imbedded in extracellular matrix, providing stability and tolerance to antibiotics and the body's innate and adaptive defences [1]. This mode of growth is an inherent feature of chronic infections and is particularly well studied for Pseudomonas aeruginosa and other Gram-negative infections [1], but also some Staphylococcus aureus infections [2]. Mycobacterial infections have also been shown to be capable of biofilm formation, most notably Mycobacterium tuberculosis (tuberculosis), which under the right conditions, can self-assemble into highly organised matrix-encapsulated biofilm [3]. Among the nontuberculous mycobacteria (NTM), Mycobacterium avium complex (MAC) and the rapidly growing mycobacteria, including MABSC, have been shown to grow as biofilms either in vitro or in environmental reservoirs $[4,5]$, but in vivo conditions have not been studied. MABSC is an emerging threat to patients with cystic fibrosis [6], who become infected at an early age and deteriorate clinically [7] as the persistent infection causes inflammation and tissue damage. We wanted to explore how MABSC grows in the antibiotic-rich, end-stage lungs of patients with cystic fibrosis. The aim was to describe the localisation and growth patterns of MABSC in vivo from freshly explanted lungs of patients with cystic fibrosis and a history of MASBC. We simultaneously performed histological and mycobacterial sampling from the same areas, from multiple pulmonary sites. Mycobacterial culture was performed by inoculation on at least one solid (Middlebrooke $7 \mathrm{H} 10$ or Löwenstein-Jensen slopes; SSI Diagnostica, Hilleroed, Denmark) and in one liquid culture medium (BACTEC 12B or MGIT; Becton Dickinson Microbiology Systems, Sparks, MD, USA), and a reverse hybridisation DNA assay was performed (InnoLiPA; Fujirebio Europe, Brondby, Denmark), as previously described [7]. Culture morphology was determined by direct visual inspection of colonies and control microscopy used Ziehl-Neelsen staining. Concomitant culture for Gram-negative and -positive bacteria was also performed. Patient files were reviewed to determine the clinical course of their end-stage lung disease and their history of other chronic bacterial infections. The NTM collection and clinical data collection was approved by the Committee on Health Research Ethics in the Capital Region of Denmark (Copenhagen, Denmark) (H-3-2012-098). The explanted lungs were collected by the transplantation team, microbiological samples were sent for NTM culture and histological samples were transferred to $4 \%$ formaldehyde before further preparation for microscopic investigation. The biopsy material was embedded in paraffin, cut into 4- $\mu \mathrm{m}$ sections and mounted on glass slides. Prior to microscopy, paraffin was removed and the tissue sections were analysed by means of conventional haematoxylin and eosin (H\&E) staining, Ziehl-Neelsen staining (acid-fast stain), auramine dye (fluorescent stain), and fluorescent in situ hybridisation (FISH) with peptide nucleic acid (PNA) probes: a uniBac probe (red) and a NTM-specific probe (green) (AdvanDx, Inc., Woburn, MA, USA). We used 4',6'-diamidino-2-phenylindole (Vector Laboratories) as a counterstain for DNA. Microscopic observations were performed with Zeiss 710 Confocal Laser Scanning Microscope (Leica Microsystems, Mannheim, Germany).

Six patients with either active $(n=5)$ or prior $(n=1)$ MABSC infection were included. None had a history of infection with other NTM species. The five patients with active MABSC disease were in intravenous or oral multidrug antimycobacterial treatment at the time of transplantation. Their clinical outcomes have previously been published [8]. MABSC was cultured from the lungs of all five patients with ongoing infection, three of which had MABSC smooth colony morphology. Morphology data was not recorded for 

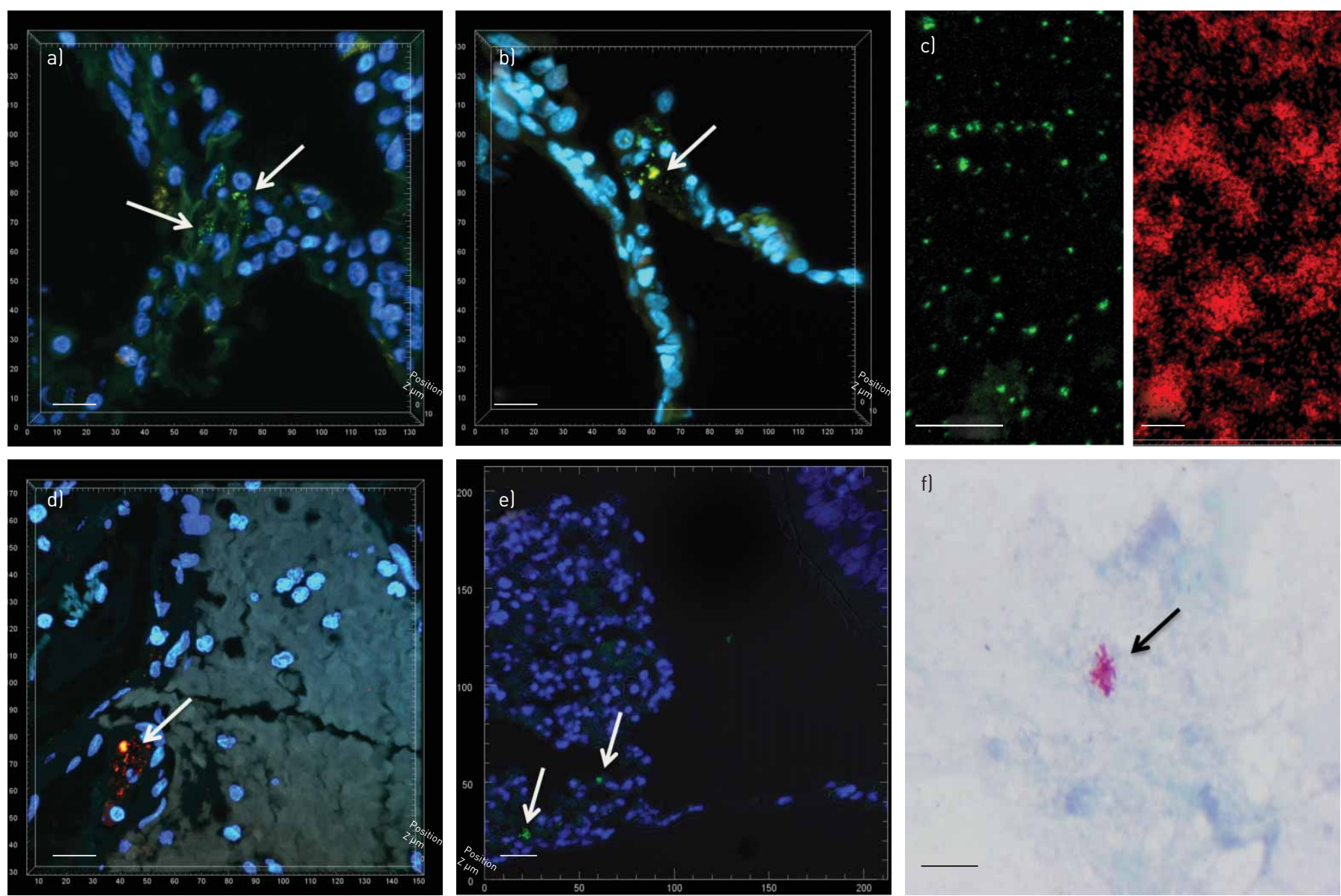

FIGURE 1 The micrographs have been obtained using a Zeiss 710 Confocal Laser Scanning Microscope with a $63 \times 1.4$ NA objective unless otherwise stated. Scale bars $=15 \mu \mathrm{m}$ unless otherwise stated. Arrows indicate bacterial biofilm. a and b) Biofilm of Mycobacterium abscessus complex (MABSC) in the pathologically thickened alveolar wall of the explanted lung of a patient with cystic fibrosis. MABSC appear bright green (fluorescein-labelled peptide nucleic acid (PNA) fluorescent in situ hybridisation (FISH) probe) and human leukocytes appear blue $\left(4^{\prime}, 6^{\prime}\right.$-diamidino-2-phenylindole (DAPI) stain). c) MABSC culture hybridised with the nontuberculous mycobacterium FISH probe (left) and the same culture stained with Ziehl-Neelsen (right), demonstrating the low yield of the PNA-FISH probe, consistent with the morphology seen in panels a and B. d) Biofilm of MABSC embedded in the alveolar wall surrounded by leukocytes (Texas Red uniBac probe and DAPI); haemorrhaging is seen in the right and central parts of the image as grey erythrocytes. e) Two small MABSC biofilm aggregates (arrows) (fluorescein-labelled PNA-FISH probe and DAPI) lodged in mucus in the conductive zone of the airways of a patient with cystic fibrosis. In the upper right part of the image, the ciliated airway epithelium can be seen. In the left side of the image, mucus with massive inflammation (dislodged from the cilia during specimen preparation) are seen. Scale bar=20 $\mu \mathrm{m}$. $\mathrm{f}$ ) Expectorated MABSC aggregate (arrow) in sputum from a patient with cystic fibrosis. Ziehl-Neelsen stain.

the other two. Confocal laser scanning microscopy was accurate in determining the presence of NTM biofilm in the lungs of four patients. No mycobacteria were seen in the patient with resolved infection. Mycobacteria were observed in sparse intra-alveolar wall microcolonies surrounded by extracellular matrix (figure 1a and b). The NTM-specific PNA-FISH probe, while highly specific, had low yield, producing suboptimal visual bacterial morphology results, but the hybridisation signals were consistent and similar staining patterns were observed in pure MABSC biofilm culture (figure 1c). Staining specimens with auramine dye gave comparable morphology results (not shown), as did hybridisation with a uniBac probe (figure 1d). The uniBac probe was used to ensure that NTM-specific staining was not autofluorescence. Micrographs of the specific region shown in figure 1d was obtained from consecutive sections, stained with either the NTM probe (not shown) or the uniBac probe (figure 1d) and similar staining results were observed. Similar signals were not visible in H\&E-stained specimens from the same area. Control hybridisation with a PNA-FISH probe specific for Stenotrophomonas maltophilia was performed in two patients with concomitant infection with this pathogen, confirming no cross-reactivity between probes. One patient was simultaneously culture positive for Achromobacter xylosoxidans and two patients for $P$. aeruginosa. Co-infection of two or more NTM species was not observed in any patient. The pattern of biofilm embedding deep in the alveolar wall was consistent in all four patients, but infrequently, single phagocytised mycobacteria and small biofilm aggregates in sputum were also observed (figure 1e). 
While it has not previously been demonstrated that pulmonary MABSC is a biofilm infection, there is abundant indirect evidence to suggest this: NTM are known to be capable of biofilm growth in the environment and in laboratory culture [4], and biofilm-like aggregates are also occasionally visible in acid-fast smears from sputum-positive NTM patients (figure 1f). MAC, the most prevalent respiratory NTM, is a notorious biofilm producer in the domestic water supply [5]. Mycobacterium ulcerans, the cause of chronic ulcers in tropical environments, is another NTM species shown to cause biofilm infection [9]. Notably, biofilm infections are not exclusively surface phenomena, as aggregates can penetrate deep into tissue, as demonstrated in chronic wounds [10]. Our finding that MABSC might be capable of biofilm growth in the human lung has important clinical implications. Biofilm growth might play a role in explaining why susceptibility testing of MABSC is so poor at predicting therapeutic success. The mechanism involved could be that the diffusion of antibiotics into the biofilm core is inhibited due to an antibiotic concentration gradient across the aggregates, sparing viable bacteria [11]. It could also be due to the heterogeneity of metabolic states in biofilms, which protects dormant bacteria, allowing adaptive evolution of the persisting bacteria [12]. Notably, recognising that MABSC is a biofilm infection gives the potential for exploring biofilm-disrupting agents. In parallel with what is seen for other chronic pulmonary biofilm infections, early antibiotic treatment may prevent biofilm formation [11]. P. aeruginosa is known to grow as biofilms, both in the mucus of the conductive airways and in the respiratory zones in patients with cystic fibrosis, and may expel nonmucoid mutants, which proliferate as planktonic cells [13]. We found MABSC in biofilm aggregates, embedded in the interstitial spaces of the respiratory zones of the lung, surrounded by inflammation. This corresponds with our finding of MABSC microaggregates in expectorated sputum of MABSC patients from all stages of the disease (figure 1f). The in vivo localisation of the biofilm in the interstitial space supports the current notion that intravenous antibiotic treatment should play a prominent role in MABSC management, as high-dose systemic antibiotics are more likely than inhaled antibiotics to reach the respiratory zones. No patients from this series received MABSC inhaled antibiotic therapy at the time of transplantation and it should be noted that the conditions in the end-stage lungs of patients with cystic fibrosis in continuous anti-MABSC treatment might not be representative of all disease stages. The reason why very limited MABSC biofilm formation was seen in sputum in the conductive zones of the examined lungs may be that mycobacteria are dormant in sputum, which is predominantly anaerobic due to the activity of polymorphonuclear leukocytes, as previously demonstrated for the aerobic S. maltophilia [14]. Our finding that the in vivo MABSC biofilms appeared to be of the smooth colony morphology type concurs with reports that this morphology is associated with persistent infection [15]. While both smooth and rough MABSC colony morphologies share the characteristics of biofilm infection (organised colony architecture), the smooth morphotype is rich in glycopeptidolipid (GPL), which might be the main constituent of the extracellular matrix [15]. The rough morphotype, a virulence factor associated with cord formation, produces much less GPL in exchange for invasive growth [15].

In summary, we have shown that patients with cystic fibrosis and chronic pulmonary MABSC infection harbour mycobacterial biofilms, embedded in the alveolar walls of their end-stage lungs.

@ERSpublications

Mycobacterium abscessus complex can establish a biofilm infection in the lung, which has implications for treatment http://ow.ly/RSjGZ

Tavs Qvist ${ }^{1,6}$, Steffen Eickhardt ${ }^{2,6}$, Kasper N. Kragh ${ }^{2}$, Claus B. Andersen ${ }^{3}$, Martin Iversen ${ }^{4}$, Niels Høiby ${ }^{2,5}$ and Thomas Bjarnsholt ${ }^{2}$

${ }^{1}$ Cystic Fibrosis Centre Copenhagen, Dept of Infectious Diseases, Rigshospitalet, Copenhagen University, Copenhagen, Denmark. ${ }^{2}$ Costerton Biofilm Centre, Dept of International Health, Immunology and Microbiology, Copenhagen University, Copenhagen, Denmark. ${ }^{3}$ Dept of Pathology, Rigshospitalet, Copenhagen University, Copenhagen, Denmark. ${ }^{4}$ Dept of Cardiology, Lung Transplantation Unit, Rigshospitalet, Copenhagen University, Copenhagen, Denmark. ${ }^{5}$ Dept of Clinical Microbiology, Rigshospitalet, Copenhagen University, Copenhagen, Denmark. ${ }^{6}$ These authors contributed equally.

Correspondence: Thomas Bjarnsholt, Costerton Biofilm Centre, Dept of International Health, Immunology and Microbiology, Panum Institute, Building 24.1, Blegdamvej 3B, DK-2200 Copenhagen N, Denmark.

E-mail: tbjarnsholt@sund.ku.dk

Received: July 092015 | Accepted after revision: Aug 192015 | First published online: Oct 222015

Conflict of interest: None declared.

\section{References}

1 Bjarnsholt T, Hoiby N, Donelli G, et al. Understanding biofilms - are we there yet? FEMS Immunol Med Microbiol 2012; 65: 125-126.

2 Archer NK, Mazaitis MJ, Costerton JW, et al. Staphylococcus aureus biofilms: properties, regulation, and roles in human disease. Virulence 2011; 2: 445-459. 
3 Kulka K, Hatfull G, Ojha AK. Growth of Mycobacterium tuberculosis biofilms. J Vis Exp 2012; 60: 3820.

4 Falkinham III JO, Falkinham JO. Surrounded by mycobacteria: nontuberculous mycobacteria in the human environment. J Appl Microbiol 2009; 107: 356-367.

5 Falkinham JO. Ecology of nontuberculous mycobacteria - where do human infections come from? Semin Respir Crit Care Med 2013; 34: 95-102.

6 Leung JM, Olivier KN. Nontuberculous mycobacteria: the changing epidemiology and treatment challenges in cystic fibrosis. Curr Opin Pulm Med 2013; 19: 662-669.

7 Qvist T, Gilljam M, Jönsson B, et al. Epidemiology of nontuberculous mycobacteria among patients with cystic fibrosis in Scandinavia. J Cyst Fibros 2015; 14: 46-52.

8 Qvist T, Pressler T, Thomsen VO, et al. Nontuberculous mycobacterial disease is not a contraindication to lung transplantation in patients with cystic fibrosis: a retrospective analysis in a Danish patient population. Transpl Proc 2013; 45: 342-345.

9 Marsollier L, Brodin P, Jackson M, et al. Impact of Mycobacterium ulcerans biofilm on transmissibility to ecological niches and Buruli ulcer pathogenesis. PLoS Pathog 2007; 3: e62.

10 Fazli M, Bjarnsholt T, Kirketerp-Møller K, et al. Nonrandom distribution of Pseudomonas aeruginosa and Staphylococcus aureus in chronic wounds. J Clin Microbiol 2009; 47: 4084-4089.

11 Høiby N, Bjarnsholt T, Moser C, et al. ESCMID guideline for the diagnosis and treatment of biofilm infections 2014. Clin Microbiol Infect 2015; 21: Suppl. 1, S1-S25.

12 Ciofu O, Nielsen TT, Jensen PØ, et al. Antimicrobial resistance, respiratory tract infections and role of biofilms in lung infections in cystic fibrosis patients. Adv Drug Deliv Rev 2015; 85: 7-23.

13 Bjarnsholt T, Jensen PO, Fiandaca MJ, et al. Pseudomonas aeruginosa biofilms in the respiratory tract of cystic fibrosis patients. Pediatr Pulmonol 2009; 44: 547-558.

14 Kolpen M, Kragh KN, Bjarnsholt T, et al. Denitrification by cystic fibrosis pathogens - Stenotrophomonas maltophilia is dormant in sputum. Int J Med Microbiol 2015; 305: 1-10.

15 Howard ST, Rhoades E, Recht J, et al. Spontaneous reversion of Mycobacterium abscessus from a smooth to a rough morphotype is associated with reduced expression of glycopeptidolipid and reacquisition of an invasive phenotype. Microbiology 2006; 152: 1581-1590.

\section{Cost-effectiveness of incorporating bedaquiline into a treatment regimen for MDR/XDR-TB in Germany}

The emergence of multi-drug-resistant (MDR) and extensively drug-resistant (XDR) tuberculosis (TB) has re-established TB as a major worldwide health concern [1-3]. Compared with conventional TB drug regimens, treatments for MDR-TB and XDR-TB are more toxic, expensive and associated with poorer outcomes [3, 4]. In Germany, 102 (2.4\%) of the 4318 TB cases reported for 2013 were MDR-TB, including 10 patients with XDR-TB [5], increasing from $54(1.8 \%)$ cases in 2011 [5, 6]. An important factor behind the increase in Germany is migration, with more cases occurring in patients from the former Soviet Union than in native Germans [5].

Bedaquiline (BDQ) is a novel treatment that may be added for 24 weeks to a World Health Organization (WHO) recommended antibiotic background regimen (BR) for the treatment of MDR-TB [7]. Wolfson et al. [8] developed a comprehensive model looking at cost-effectiveness of BDQ plus BR (BDQ+BR) in the UK, over a substantially longer time horizon than a previously published WHO analysis $[8,9]$.

In this study, we report findings from an adapted version examining cost-effectiveness in the German healthcare system, where typically routine hospitalisation of patients with MDR-TB is carried out. A previous study of patients with MDR- TB in Germany found the total cost of treating to be $€ 64429.23$ [10].

After adjustment to the perspective of the German Statutory Health Insurance (SHI) system, a cohort-based Markov model was used to estimate the cost-effectiveness of BDQ as an add-on for 24 weeks to a weighted antibiotic BR containing pyrazinamide, one injectable, one fluoroquinolone and one WHO group 4 agent, taking into account different strain resistance patterns as described in the C208 trial and following local guidelines $[4,7,11]$. This regimen of $\mathrm{BDQ}+\mathrm{BR}$ was then compared with $\mathrm{BR}$ alone for the treatment of MDR- and XDR-TB. Comparison of BDQ with Delamanid, an alternative treatment option that was introduced later [12], was outside the focus of our analysis. Costs and effectiveness were discounted at an annual rate of 3\% [13]. The time horizon for the model was 10 years. The model state structure was designed to reflect initial clinical care in patients with TB. 